\title{
A swirling flare-related EUV jet ${ }^{\star}$
}

\author{
Q. M. Zhang and H. S. Ji
}

\author{
Key Laboratory for Dark Matter and Space Science, Purple Mountain Observatory, CAS, 210008 Nanjing, PR China \\ e-mail: zhangqm@pmo.ac.cn
}

Received 5 September 2013 / Accepted 4 December 2013

\begin{abstract}
Aims. We report our observations of a swirling flare-related extreme-ultraviolet (EUV) jet on 2011 October 15 at the edge of NOAA active region 11314 .

Methods. We used the multiwavelength observations in the EUV passbands from the Atmospheric Imaging Assembly (AIA) aboard the Solar Dynamics Observatory (SDO). We extracted a wide slit along the jet axis and 12 thin slits across its axis to investigate the longitudinal motion and transverse rotation. We also used data from the Extreme-Ultraviolet Imager (EUVI) aboard the Solar TErrestrial RElations Observatory (STEREO) spacecraft to investigate the three-dimensional (3D) structure of the jet. Ground-based $\mathrm{H} \alpha$ images from the El Teide Observatory, a member of the Global Oscillation Network Group (GONG), provide a good opportunity to explore the relationship between the cool surge and the hot jet. Line-of-sight magnetograms from the Helioseismic and Magnetic Imager (HMI) aboard SDO enable us to study the magnetic evolution of the flare/jet event. We carried out potential-field extrapolation to calculate the magnetic configuration associated with the jet.

Results. The onset of jet eruption coincided with the start time of the $\mathrm{C} 1.6$ flare impulsive phase. The initial velocity and acceleration of the longitudinal motion were $254 \pm 10 \mathrm{~km} \mathrm{~s}^{-1}$ and $-97 \pm 5 \mathrm{~m} \mathrm{~s}^{-2}$, respectively. The jet presented helical structure and transverse swirling motion at the beginning of its eruption. The counter-clockwise rotation slowed down from an average velocity of $\sim 122 \mathrm{~km} \mathrm{~s}{ }^{-1}$ to $\sim 80 \mathrm{~km} \mathrm{~s}^{-1}$. The interwinding thick threads of the jet untwisted into multiple thin threads during the rotation that lasted for one cycle with a period of $\sim 7 \mathrm{~min}$ and an amplitude that increases from $\sim 3.2 \mathrm{Mm}$ at the bottom to $\sim 11 \mathrm{Mm}$ at the upper part. Afterwards, the curtain-like leading edge of the jet continued rising without rotation, leaving a dimming region behind, before falling back to the solar surface. The appearance/disappearance of dimming corresponded to the longitudinal ascending/descending motions of jet. Cospatial $\mathrm{H} \alpha$ surge and EUV dimming imply that the dimming resulted from the absorption of hot EUV emission by the cool surge. The flare/jet event was caused by continuous magnetic cancellation before the start of the flare. The jet was associated with the open magnetic fields at the edge of AR 11314.
\end{abstract}

Key words. Sun: corona - Sun: oscillations - Sun: flares

\section{Introduction}

There are various jet-like activities in the solar atmosphere, such as spicules (De Pontieu et al. 2004), chromospheric jets (Shibata et al. 2007; Nishizuka et al. 2008; Liu et al. 2009, 2011b; Singh et al. 2012), H $\alpha$ surges (Schmieder et al. 1995; Zhang et al. 2000; Liu \& Kurokawa 2004; Jiang et al. 2007), extreme-ultraviolet (EUV) jets (Chae et al. 1999; Nisticò et al. 2009; Moschou et al. 2012), and soft X-ray (SXR) jets (Shibata et al. 1992; Savcheva et al. 2007). Most of the coronal jets seen in EUV and SXR are associated with flares, microflares, or bright points at their footpoints (Ji et al. 2008; Zhang et al. 2012; Zhang \& Ji 2013). The typical height of jets is $10-400 \mathrm{Mm}$, the width is $5-100 \mathrm{Mm}$, the longitudinal velocity is $10-1000 \mathrm{~km} \mathrm{~s}^{-1}$ with an average value of $\sim 200 \mathrm{~km} \mathrm{~s}^{-1}$, and the kinetic energy is on the order of $10^{25}-10^{28}$ erg (Shimojo et al. 1996). Coronal jets are formed in coronal holes (Wang et al. 1998; Cirtain et al. 2007; Culhane 2007; Patsourakos et al. 2008) or at the edge of active regions (Kim et al. 2007; Guo et al. 2013) in the presence of open magnetic fields. Particles are accelerated and ejected into the interplanetary space during the reconnection, generating Type III radio bursts (Krucker et al. 2011; Glesener et al. 2012). Coronal jets are believed to be heated by magnetic reconnection between

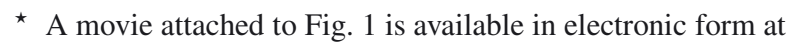
http://www . aanda.org emerging flux and the pre-existing magnetic fields (Yokoyama \& Shibata 1996; Moreno-Insertis et al. 2008; Török et al. 2009; Jiang et al. 2012; Moreno-Insertis \& Galsgaard 2013; Pontin et al. 2013). Moore et al. (2010) classified jets into two types, standard jets and blowout jets; the latter features blowout eruption of the base arch's core field.

Apart from the ordinary collimated motions, jets occasionally exhibit helical structure and untwisting motions (Liu et al. 2011a; Shen et al. 2011; Chen et al. 2012; Hong et al. 2013; Schmieder et al. 2013). The amplitude of transverse rotations ranges from 2 to $10 \mathrm{Mm}$. The periods are on the order of 4-9 min. The transverse velocities $\left(10-150 \mathrm{~km} \mathrm{~s}^{-1}\right)$ are slightly lower than their longitudinal velocities along the jet axis. The untwisting motions were previously interpreted as the release of magnetic helicity during the reconnection between a twisted bipole and open fields (Shibata \& Uchida 1986; Canfield et al. 1996; Jibben \& Canfield 2004). Pariat et al. (2009, 2010) numerically simulated the formation of untwisting jets as a result of continuous pumping of magnetic free energy and helicity into the corona from the photosphere, which is interpreted as upward propagation of torsional Alfvén waves at a speed of hundreds of $\mathrm{km} \mathrm{s}^{-1}$. Numerous flare-related jets and rotating jets have been observed. In this paper, we report the helical structure and swirling motion of a flare-related EUV jet observed by the Atmospheric Imaging Assembly (AIA; Lemen et al. 2012) aboard the Solar Dynamics Observatory (SDO) spacecraft. In 
Sect. 2, we describe the multiwavelength data analysis. The results are shown in Sect. 3. Discussion and summary are presented in Sect. 4.

\section{Data analysis}

The SDO/AIA instrument has high cadence and resolution in the seven EUV wavelengths $(94,131,171,193,211,304$, and $335 \AA$ ). A C1.6 flare and the accompanying jet at the edge of NOAA active region 11314 was observed by AIA during 11:00-14:00 UT on 2011 October 15. The full-disk level_1 fits data were calibrated using the standard program aia_prep.pro in the Solar Software. Image coalignments between the EUV passbands were performed using the crosscorrelation method after selecting a bright feature (e.g., active region). The accuracy of coalignments is 1 '. 2 . In order to investigate the three-dimensional (3D) structure of the jet, we checked data from the Extreme-Ultraviolet Imager (EUVI) in the Sun Earth Connection Coronal and Heliospheric Investigation (SECCHI; Howard et al. 2008) package aboard the Solar TErrestrial RElations Observatory (STEREO; Kaiser 2005). The orbits of the twin satellites (A and B) drifted as time goes by, so that they had separation angles of $\sim 105^{\circ}$ and $\sim 99^{\circ}$ with the Sun-Earth connection on that day. Combined with SDO, the perspectives from STEREO provide an excellent opportunity to get a full view of solar eruptions. The $195 \AA$ raw data were calibrated using the program secchi_prep.pro. The deviation of the STEREO N-S direction from the solar rotation axis was corrected. After checking the EUV observation during that time, we found that the jet was visible only in the field of view of STEREO-B, but still partly blocked by the solar western limb. To explore the relationship between the hot jet and cool surge, we examined the gound-based $\mathrm{H} \alpha$ observation from the El Teide Observatory, which is a member of the Global Oscillation Network Group (GONG). The $\mathrm{H} \alpha$ images were coaligned with the AIA $304 \AA$ images owing to their lower formation heights. Unfortunately, there was a data gap during 11:57-13:12 UT when the flare occurred. The line-ofsight (LOS) magnetograms from the Helioseismic and Magnetic Imager (HMI; Scherrer et al. 2012) aboard SDO were used to study the temporal evolution of magnetic fields in the photosphere. The magnetograms were coaligned with the $\mathrm{H} \alpha$ images according to the position of the sunspot. Moreover, we carried out potential-field extrapolation to calculate the 3D magnetic configuration of the jet. The E-W and N-S scopes of the magnetogram for extrapolation were $886 . ' 8$ and 706.2 so that flux balance was guaranteed. Soft X-ray light curves from the Geostationary Satellite (GOES) were used to investigate the evolution of $\mathrm{C} 1.6$ flare. The observing parameters are summarized in Table 1, including the instruments, wavelengths $(\lambda)$, observing times, cadences, and pixel sizes.

\section{Results}

In Fig. 1, the three snapshots of AIA $193 \AA$ images represent the three phases of flare: pre-flare phase (a), impulsive phase (b), and main phase (c), respectively. In the pre-flare phase, there was very weak brightening at the flare site in the absence of jet. In the impulsive phase, the area and total emission of flare quickly increased and reached maximum. The helical structure of jet was composed of two interwinding threads (labeled T1 and T2) that started counter-clockwise rotation perpendicular to its axis. In the main phase, the total emission of flare decreased
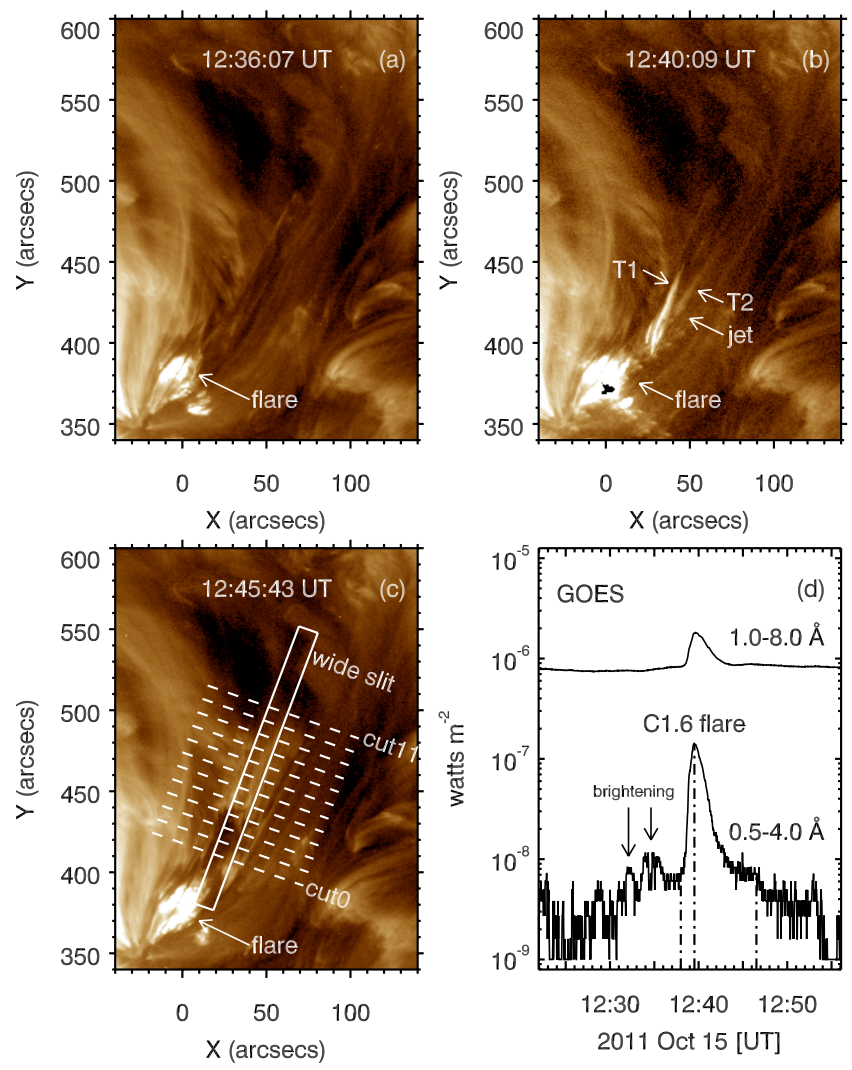

Fig. 1. (Movie online) a)-c) Three snapshots of the $193 \AA$ images. The white arrows point to the flare and jet. In panel b), the two thick threads of the jet are labeled "T1" and "T2". In panel c), the solid box and dashed lines represent the wide slit and 12 narrow slits (cut0-cut11), respectively. d) GOES light curves in 1-8 $\AA$ (upper) and 0.5-4.0 $\AA$ (lower), featuring the C1.6 flare and weak precursor brightenings. The dash-dotted lines denote the start (12:38:00 UT) and peak (12:39:30 UT) of the flare impulsive phase and end (12:46:30 UT) of the main phase. The temporal evolution is shown in a movie with a larger field of view available in the online edition.

Table 1. Description of the observational parameters.

\begin{tabular}{lcccc}
\hline \hline Instrument & $\begin{array}{c}\lambda \\
(\AA)\end{array}$ & $\begin{array}{c}\text { Time } \\
(\mathrm{UT})\end{array}$ & $\begin{array}{c}\text { Cadence } \\
(\mathrm{s})\end{array}$ & $\begin{array}{c}\text { Pixel size } \\
(\operatorname{arcsec})\end{array}$ \\
\hline AIA & $94-335$ & $11: 00-14: 00$ & 12 & 0.6 \\
HMI & - & $11: 00-14: 00$ & 45 & 0.6 \\
EUVI & 195 & $11: 00-14: 00$ & 300 & 1.6 \\
EI Teide & 6563 & $11: 10-11: 57$ & 60 & 1.0 \\
EI Teide & 6563 & $13: 12-14: 00$ & 60 & 1.0 \\
GOES & $0.5-4.0$ & $11: 00-14: 00$ & 3 & - \\
GOES & $1.0-8.0$ & $11: 00-14: 00$ & 3 & - \\
\hline
\end{tabular}

significantly. The two compact threads untwisted into many thin threads, suggesting the multistrand nature of the jet. Afterwards, the jet experienced a curtain-like eruption along its axis. To investigate the longitudinal and transverse motions, we extracted a wide slit along the axis and 12 narrow slits (from cut 0 to cut11) perpendicular to the axis, which is shown in Fig. 1c. The wide slit (solid box) is $12^{\prime \prime}$ in width and $182^{\prime \prime}$ in length. The narrow slits (dashed lines) are 96" in length. In Fig. 1d, we plot the light curves in 1.0-8.0 $\AA$ (upper) and 0.5-4.0 $\AA$ (lower). The impulsive phase of the flare started at 12:38:00 UT and peaked at 12:39:30 UT, and the main phase ended at 12:46:30 UT, which is marked by the dash-dotted lines. 

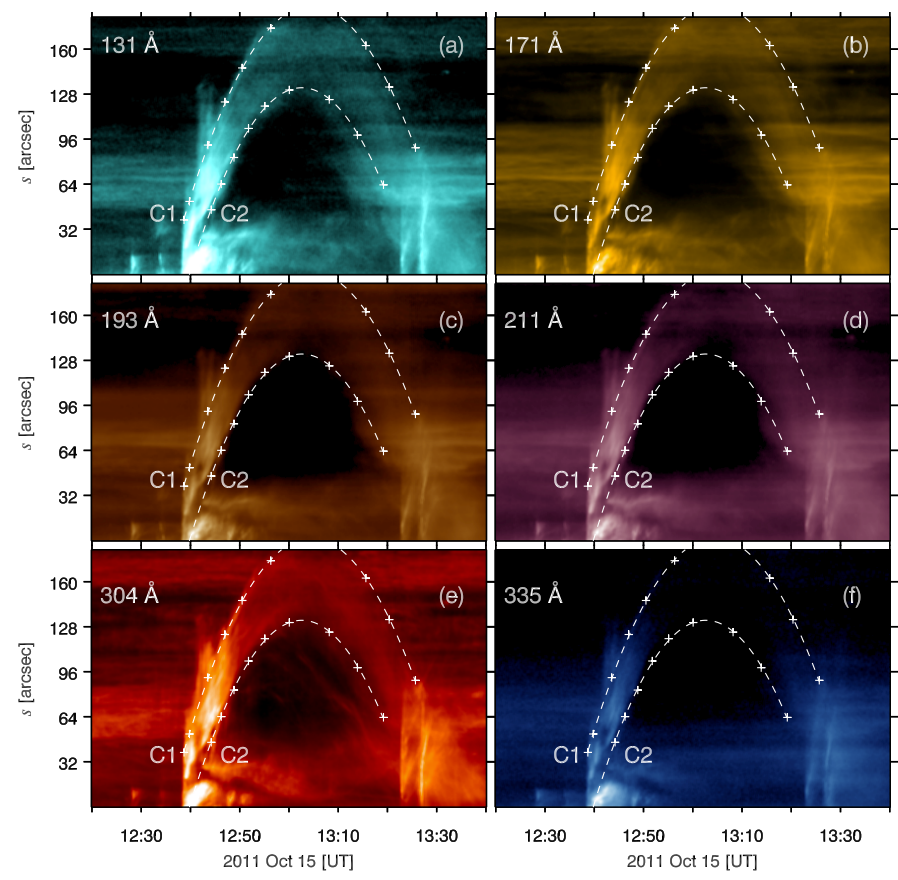

Fig. 2. a)-f) Time-slice diagrams of the wide slit in six of the EUV passbands. $s=0^{\prime \prime}$ and $182^{\prime \prime}$ in the $y$-axis represent the bottom-left and topright endpoints of the slit. The two dashed lines stand for the outer (C1) and inner (C2) boundaries of the parabolic trajectory.

In Fig. 2, the time-slice diagrams of the wide slit in six of the EUV passbands (131, 171, 193, 211, 304, and $335 \AA$ ) illustrate the longitudinal motion of the jet along its axis. The jet underwent a parabolic trajectory during 12:38-13:25 UT. We outline the outer and inner boundaries of the trajectory with two curves (C1 and $\mathrm{C} 2$ ) that are fitted with a quadratic function:

$s=s_{0}+v_{\|}\left(t-t_{0}\right)+\frac{1}{2} g_{\|}\left(t-t_{0}\right)^{2}$,

where $t_{0}=12: 38 \mathrm{UT}$, the initial velocity $v_{\|}=264$ and $244 \mathrm{~km} \mathrm{~s}^{-1}$, the acceleration $g_{\|}=-103$ and $-92 \mathrm{~m} \mathrm{~s}^{-2}$ for $\mathrm{C} 1$ and $\mathrm{C} 2$, respectively. Combining the two curves, we obtain $v_{\|}=254 \pm 10 \mathrm{~km} \mathrm{~s}^{-1}$ and $g_{\|}=-97 \pm 5 \mathrm{~m} \mathrm{~s}^{-2}$.

Figure 3 displays the time-slice diagrams of ten narrow slits (cut2-cut11) in $193 \AA$. The value $s$ in the $y$-axis of each panel signifies the distance from the top-left endpoints of slits. The middle of the $y$-axis $\left(s=48^{\prime \prime}\right)$ corresponds to the position of the jet axis. It is clear that the jet rotated leftwards during 12:40-12:44 UT, which is illustrated by the solid oblique lines whose slopes stand for the transverse velocities. The jet rotated rightwards during 12:44-12:48 UT, which is also illustrated by the oblique lines. We calculated the jet velocities from the 34 labeled lines and show the histogram in Fig. 4 where negative/positive values represent leftward/rightward rotation. It is revealed that the rotation slowed down from an average leftward speed of $\sim 122 \mathrm{~km} \mathrm{~s}^{-1}$ to an average rightward speed of $\sim 80 \mathrm{~km} \mathrm{~s}^{-1}$. The rotation lasted for only one cycle whose pe$\operatorname{riod}(427 \mathrm{~s})$ and twofold amplitude $(22 \mathrm{Mm})$ are marked in the time-slice diagram of cut5, i.e., the seventh row of Fig. 3 . The near-simultaneous reversals of direction around 12:44 UT indicate that the whole jet rotated in phase.

After the transverse rotation, the helical jet became curtainlike and continued rising in the longitudinal direction, leaving behind a dimming, which is manifested by the central dark regions in all panels of Fig. 3. We label the dimming region with

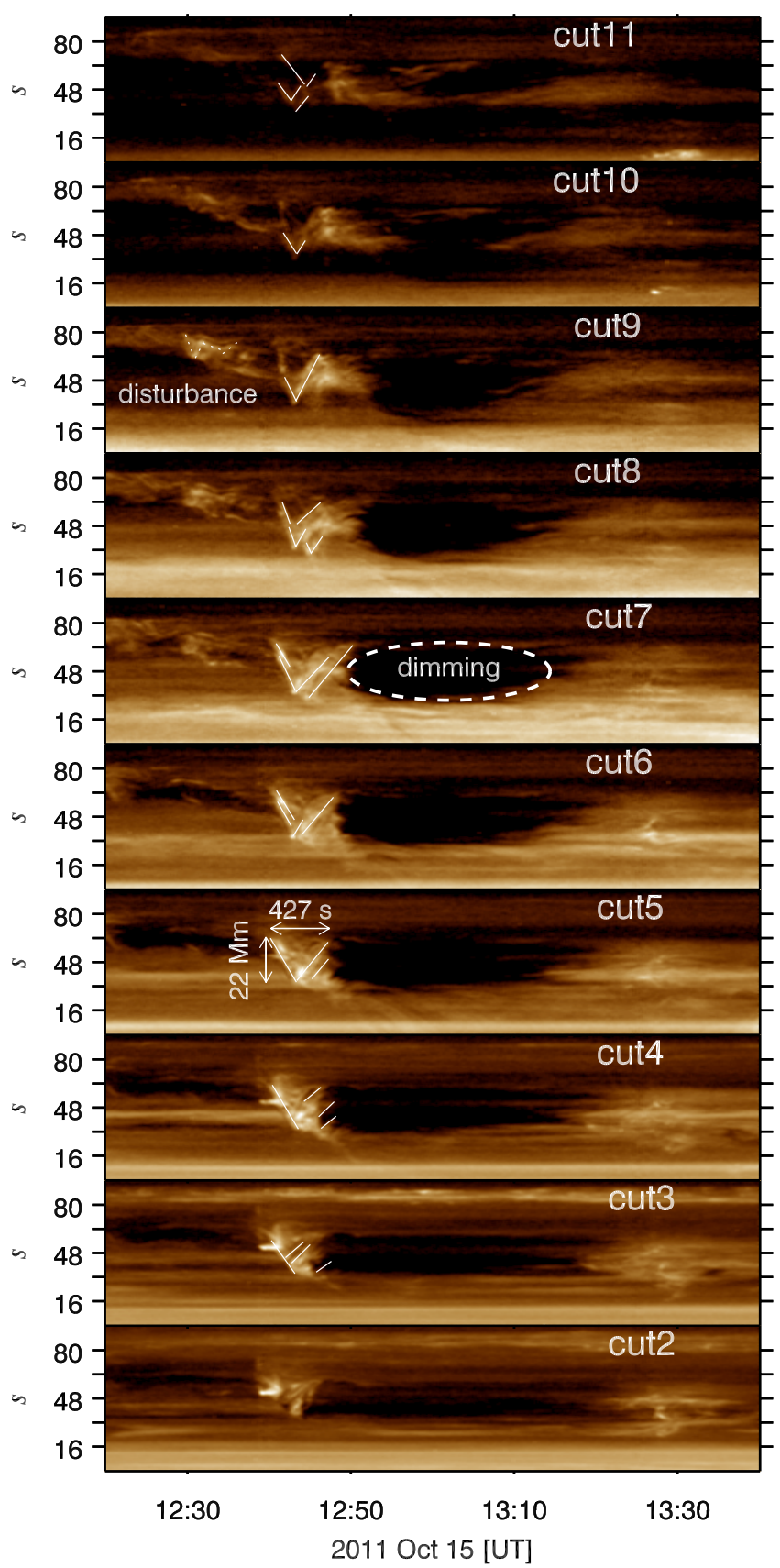

Fig. 3. Time-slice diagrams of the ten narrow slits (cut2-cut11) in $193 \AA$ A. $s=0$ " and $96^{\prime \prime}$ in the $y$-axis signify the top-left and bottomright endpoints of slits. The short solid lines denote the 34 manually selected bright features signifying the leftward and rightward transverse rotation of the jet. The short dotted lines in the third panel from the top denote the four manually selected bright features signifying the precursor disturbances adjacent to the jet axis. The dashed ellipse in the fifth panel outlines the boundary of dimming. The horizontal and vertical double-headed arrows in the seventh panel measure the period (427 s) and double amplitude (22 Mm) of rotation.

a dashed ellipse in the fifth row of Fig. 3. The appearances of dimming occurred successively from the lower to upper parts of the jet. The disappearances of dimming took place successively, however, from the upper to lower parts of the jet. Combining the longitudinal evolutions of the jet in Fig. 2, we found that the appearance/disappearance of dimming coincided with the longitudinal ascending/descending motions. 


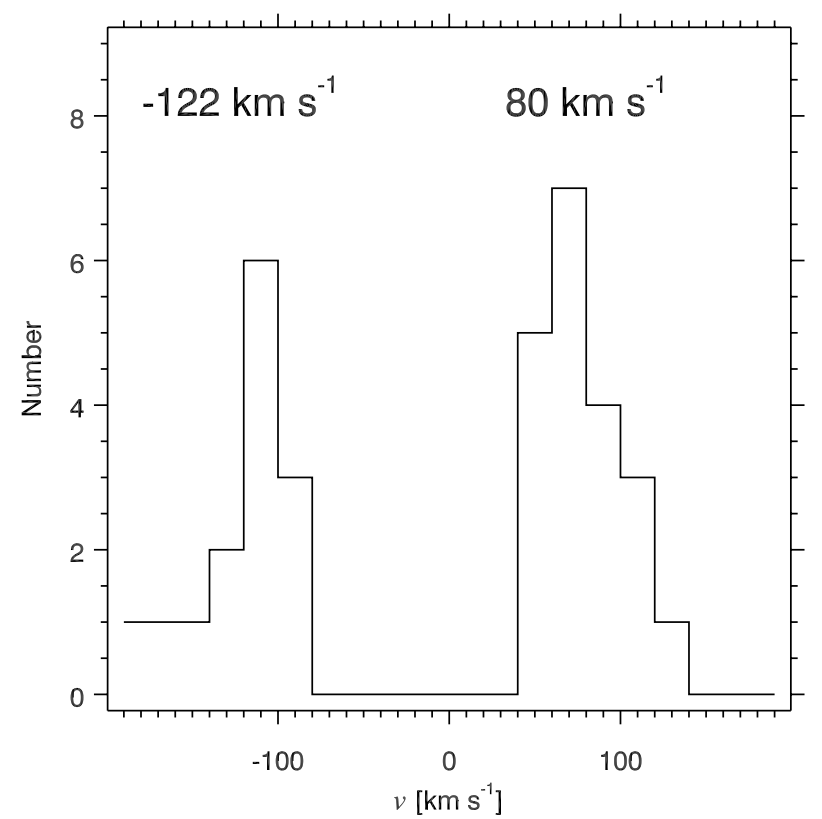

Fig. 4. Histogram of the transverse velocities of the jet rotation. Negative/positive values suggest leftward/rightward motions. The average leftward speed $\left(-122 \mathrm{~km} \mathrm{~s}^{-1}\right)$ and average rightward speed $\left(80 \mathrm{~km} \mathrm{~s}^{-1}\right)$ of the jet rotation are labeled.

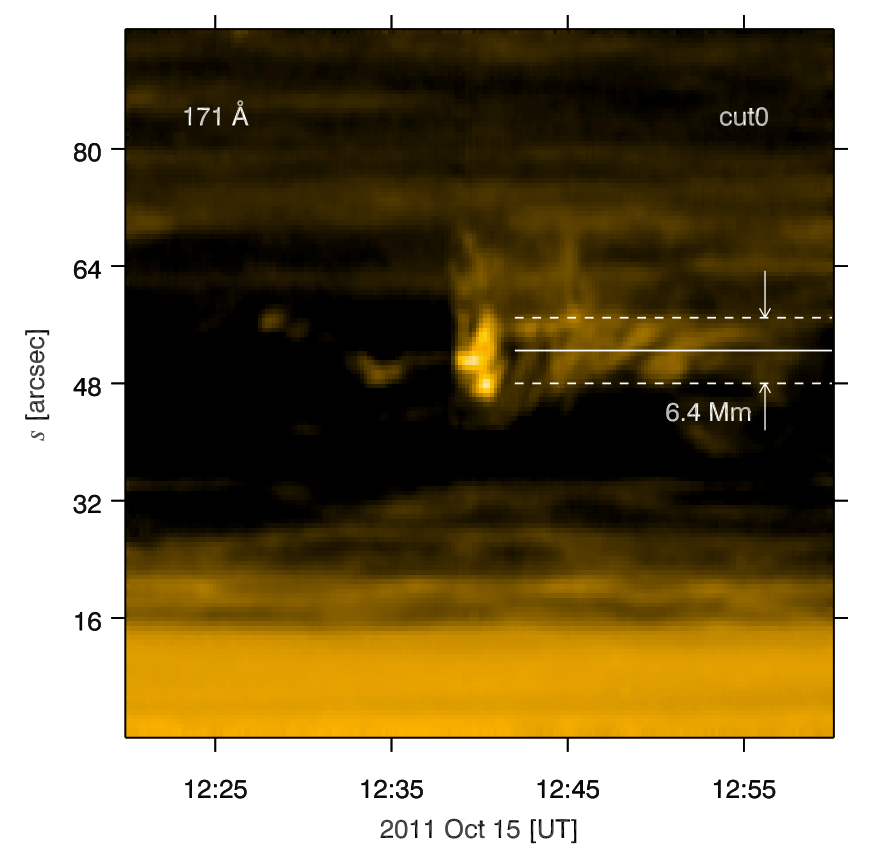

Fig. 5. Time-slice diagram of cut 0 near the bottom of the jet in $171 \AA$. The solid and dashed lines mark the center and boundaries of the helical feature that indicates swirling motion of the jet. The distance between the dashed lines $(6.4 \mathrm{Mm})$ equals double the amplitude of the jet rotation at cut 0 .

Figure 5 shows the time-slice diagram of cut 0 that is close to the base of jet. The helical feature in the diagram suggests transverse rotation during $\sim 12: 42-12: 55$ UT. According to the handedness of the feature, we conclude that the jet rotated counter-clockwise with respect to its axis. We extracted the intensity profile along the center of the helical pattern, which is labeled with a solid line bounded by two dashed lines. The distance $(6.4 \mathrm{Mm})$ between the dashed lines equals double the amplitude of rotation at cut 0 .
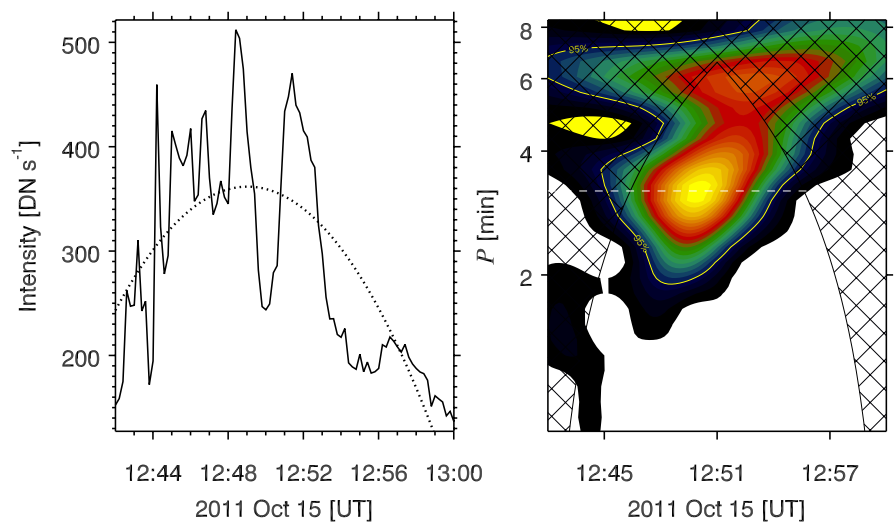

Fig. 6. Left panel: intensity profile (solid) and its fitted trend (dotted) along the solid line in Fig. 5. Right panel: wavelet transform of the trend-removed intensity profile. The white dashed line signifies the pe$\operatorname{riod}(\sim 192$ s) with maximum power.

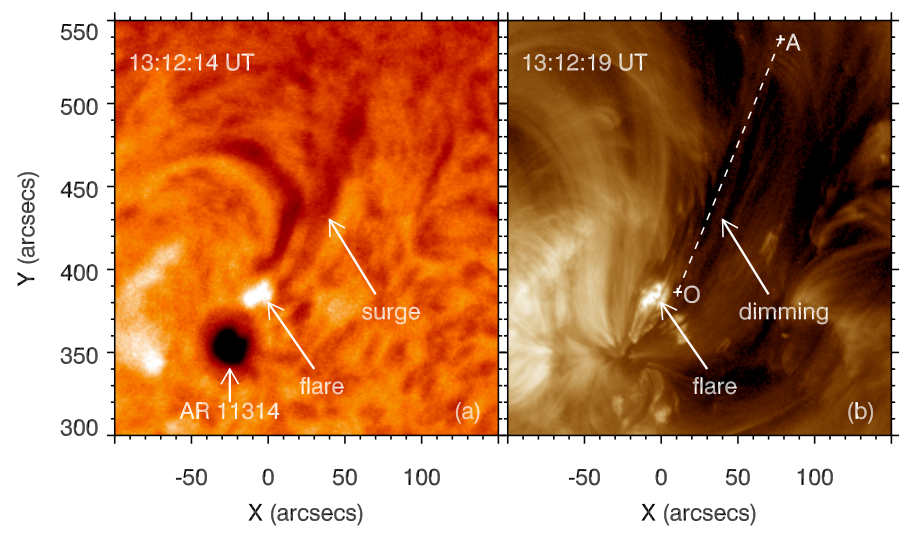

Fig. 7. Left panel: $\mathrm{H} \alpha$ image at 13:12:14 UT featuring the AR 11314, flare, and cool surge as indicated by the white arrows. Right panel: AIA $193 \AA$ A image at 13:12:19 UT featuring the flare and dimming following the leading edge of the jet as indicated by the white arrows. The dashed line ("OA") is used to measure the true height of jet.

In the left panel of Fig. 6, the intensity profile and its quadratic-fitted trend are plotted in solid and dotted lines. It is evident that the intensity profile presents quasi-periodic oscillation. The result of the wavelet transform of the trend-removed profile is shown in the right panel where the bright yellow region at the center suggests quasi-periodicity of the curve with period of $\sim 192 \mathrm{~s}$. However, considering that the jet was composed of two compact interwinding threads at its bottom (T1 and T2 in Fig. 1b), the true period of rotation should be $\sim 384 \mathrm{~s}$, which is slightly shorter than the measured value of $427 \mathrm{~s}$ in the seventh panel of Fig. 3, implying that the jet rotates faster at the lower part than the upper part. The amplitude of rotation at the base of the jet $(\sim 3.2 \mathrm{Mm})$ accounts for $30 \%$ of the value at the upper part.

Figure 7 shows the $\mathrm{H} \alpha$ and AIA $193 \AA$ images at 13:12 UT. It is clear that the cool surge seen in the $\mathrm{H} \alpha$ passband is cospatial with the dimming region following the leading edge of the jet. The surge was visible until 13:17 UT. Unfortunately, there was a data gap before 13:12 UT. We are unable to investigate the temporal relation between the jet and surge during the flare.

Compact solar flares are usually associated with magnetic flux emergence or cancellation. To understand the cause of flare/jet events, we checked the HMI LOS magnetograms. In Fig. 8, we display four magnetograms around the sunspot of 


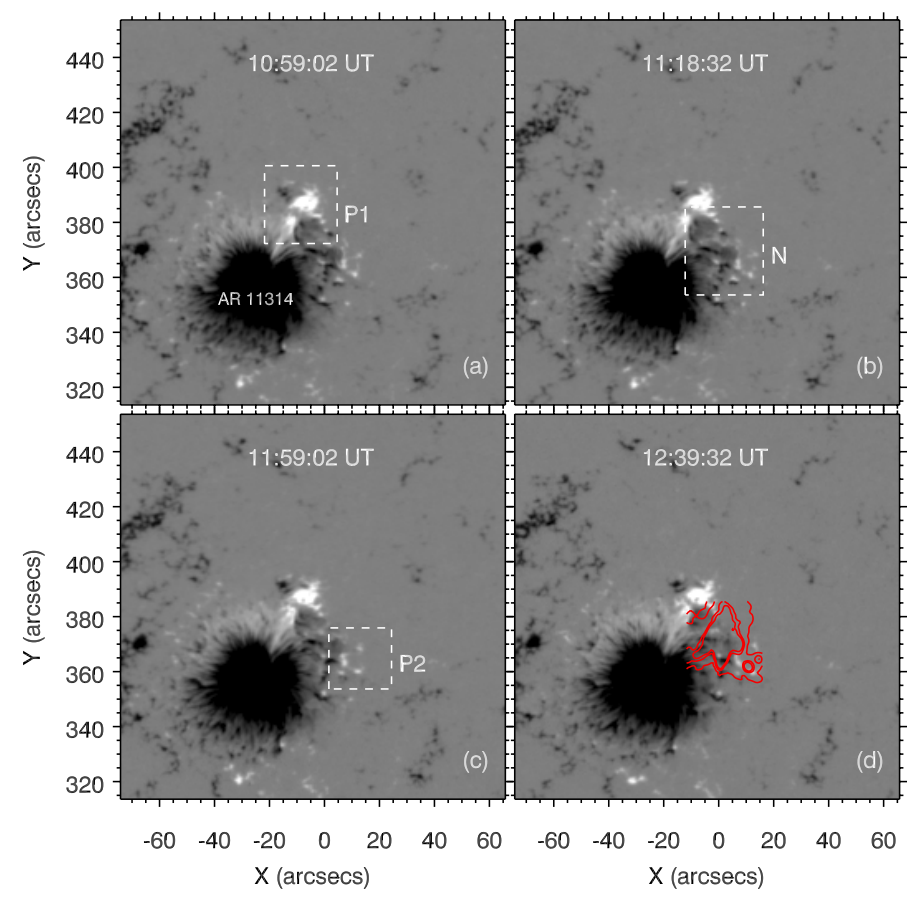

Fig. 8. HMI LOS magnetograms at 10:59:02 UT a), 11:18:32 UT b), 11:59:02 UT c), and 12:39:32 UT d). P1 and P2 with positive polarity are indicated by the white dashed boxes in panels a) and c). $N$ with negative polarity is indicated by the white dashed box in panel b). The red lines in panel d) represent the intensity contour of the flare shown in the top-left panel of Fig. 10.

AR 11314. The flare took place near the northwest boundary of the strong sunspot with negative magnetic polarity (Fig. 8d). We label the negative-polarity region around the sunspot with "N" (Fig. 8b) and the neighbouring positive-polarity regions with "P1" (Fig. 8a) and "P2" (Fig. 8c). After viewing the movie of the magnetograms, we discovered magnetic cancellation before the flare. The temporal evolutions of magnetic flux of the three regions are demonstrated in Fig. 9 where $\Phi_{\mathrm{P} 1}$, $\Phi_{\mathrm{P} 2}$, and $\Phi_{\mathrm{N}}$ represent the total positive magnetic flux of P1, the positive flux of $\mathrm{P} 2$, and the negative flux of $\mathrm{N}$. It is evident that all three polarities underwent continuous cancellation with small-scale fluctuations before the onset of the jet and flare impulsive phase at 12:38 UT. Using linear curve-fitting, we derived the cancellation rate for the three polarities, being $\mathrm{d} \Phi_{\mathrm{P} 1} / \mathrm{d} t=-1.1 \times 10^{17} \mathrm{Mx} \mathrm{h}^{-1}, \mathrm{~d} \Phi_{\mathrm{P} 2} / \mathrm{d} t=-4.6 \times 10^{16} \mathrm{Mx} \mathrm{h}^{-1}$, and $\mathrm{d} \Phi_{\mathrm{N}} / \mathrm{d} t=-8.0 \times 10^{17} \mathrm{Mx} \mathrm{h}^{-1}$, respectively. The magnetic fluxes of P2 and $\mathrm{N}$ kept on cancelling after the flare, while the flux of P1 fluctuated and kept near-constant. The observations of jets and surges as a result of flux emergence and cancellation have been reported (Liu \& Kurokawa 2004; Jiang et al. 2007; Chifor et al. 2008).

To derive the $3 \mathrm{D}$ magnetic configuration of the flare-related jet, we performed potential-field extrapolation. In the top-left panel of Fig. 10, we show the AIA $193 \AA$ image at 12:39:31 UT superposed by the HMI magnetogram contours at the same time, where the red/blue lines stand for positive/negative polarities. It features the bright compact flare and jet in the northwest direction. In the bottom-left panel of Fig. 10, we display the top-view of magnetic configuration, where the grayscale image represents the magnetogram and the red/blue lines denote the closed/open magnetic field lines. It is obvious that the jet direction is very consistent with the orientation of the projected open field lines.

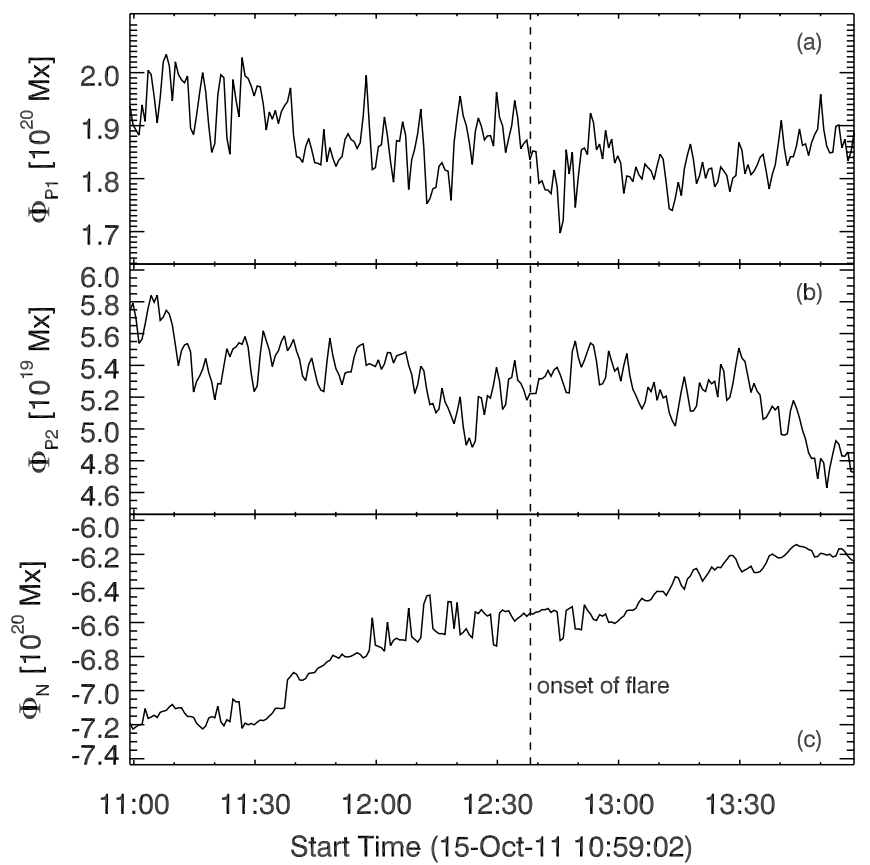

Fig. 9. Temporal evolutions of the magnetic fluxes of $\mathrm{P} 1 \mathbf{a}$ ), $\mathrm{P} 2 \mathbf{b}$ ), and $\mathrm{N}$ c). The dashed line denotes the start of the jet and flare impulsive phase.
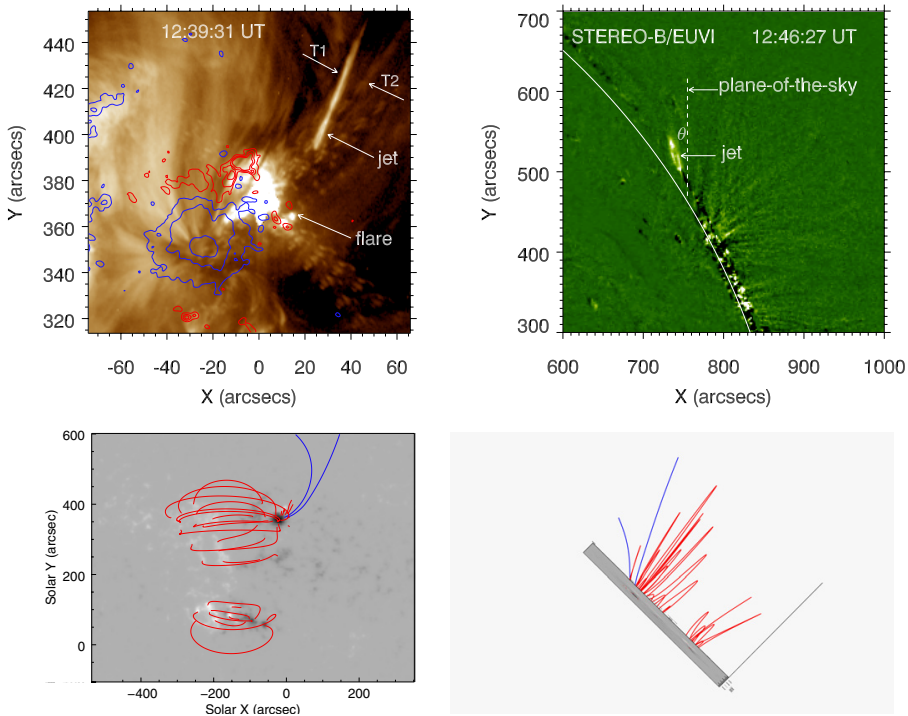

Fig. 10. Top left: AIA $193 \AA$ image at 12:39:31 UT that features the jet threads (T1 and T2) and flare as indicated by the white arrows. The red/blue lines stand for the contours of positive and negative polarities of the HMI LOS magnetogram at 12:39:32 UT. Top right: runningdifference image of STEREO-B/EUVI in $195 \AA$ at 12:46:27 UT, showing the top segment of the jet. The dashed line stands for the projection of the plane-of-the-sky on the EUV image. The angle included between the jet and plane-of-the-sky is labeled with $\theta$. Bottom panels: top view (left) and side view (right) of the 3D magnetic field lines. The red/blue lines represent closed/open magnetic field lines.

In the top-right panel of Fig. 10, we display the EUVI running-difference image at 12:46:27 UT in $195 \AA$. Only the top segment of the jet was observed while the lower segment of jet and flare were blocked. The included angle between the jet and the projection of the plane-of-the-sky on the EUVI image (dashed line) is denoted with $\theta$ which measures $11.3^{\circ}$. In the bottom-right panel of Fig. 10, we show the side view of the magnetic field lines from the STEREO-B viewpoint. It is obvious 
that the direction of jet is also consistent with the direction of the open fields.

Since we have two perspectives from which to view the flarerelated jet, one from SDO/AIA and the other from STEREO$\mathrm{B} / \mathrm{EUVI}$, we can take the projection effect into account to estimate the true height and speed. In the right panel of Fig. 7, we label the bottom and leading edge of jet with "O" and "A", whose distance equals to the apparent height of the jet, i.e., $120.7 \mathrm{Mm}$. Considering the angular derivation $(\theta)$ of the jet from the planeof-the-sky, the true height and initial velocity are estimated to be $122.7 \mathrm{Mm}$ and $258 \pm 10 \mathrm{~km} \mathrm{~s}^{-1}$.

\section{Discussion and summary}

The rising and subsequent falling of jets and surges along their axis have been extensively reported. Roy (1973) found that the acceleration of falling material of $\mathrm{H} \alpha$ surges was less than the solar free-fall acceleration $\left(g_{\odot}\right)$. Liu et al. (2009) found that the effective gravitational acceleration of the $\mathrm{Ca}$ II $\mathrm{H}$ jet had a mean value of $141 \mathrm{~m} \mathrm{~s}^{-2}$. Shen et al. (2011) discovered an even smaller value $\left(26 \mathrm{~m} \mathrm{~s}^{-2}\right)$ that was interpreted as the projection effect or damping effect of the background magnetic fields. For our case, the parallel gravitational acceleration $\left(g_{\|}\right)$accounts for $\frac{1}{3} g_{\odot}-\frac{1}{2} g_{\odot}$ in the solar atmosphere. We propose that the Lorentz force of twisted jet threads (T1 and T2 in Fig. 1b) serves as an upward force against gravity.

Coronal mass ejections (Chen 2011; Cheng et al. 2011) are often accompanied by EUV waves and the following dimming that was interpreted as density depletion (Chen et al. 2002, 2010). Such dimmings accompanying EUV jets have rarely been reported. Shen et al. (2011) observed a cavity obvious in $304 \AA$ but undetectable in $193 \AA$, suggesting the cool temperature of the cavity. Lee et al. (2013) discovered a fast jet-associated EUV dimming that is explained by Alfvénic waves initiated by reconnection in the upper chromosphere. In our study, the dimming following the leading edge of the jet was detectable in all the EUV passbands of AIA. The appearance/disappearance of dimming coincided with the longitudinal rising/falling motions of the jet. It is shown in Fig. 7 that the dimming is cospatial with the $\mathrm{H} \alpha$ surge, indicating that the dimming resulted from the absorption of hot EUV emission by the cool surge. The disappearance of surge at $\sim 13: 18$ UT coincided with the disappearance of dimming around 13:20 UT. Yokoyama \& Shibata (1996) performed magnetohydrodynamic numerical simulations to explain the generation of hot jets and cool surges during the magnetic reconnection between the emerging magnetic flux and the preexisting fields of opposite polarity. The jet and surge are adjacent in the 2D simulation. However, if we observe the eruption from an appropriate viewpoint, the jet would be blocked by the foreground surge, leading to the absorption of EUV emission by the $\mathrm{H} \alpha$ surge at a lower speed.

The helical structure and untwisting motions of EUV jets have been extensively investigated since the launch of highresolution space telescopes (e.g., SDO). These motions were often explained by the release of accumulated magnetic helicity into the upper solar atmosphere. The jet we observed underwent counter-clockwise rotation at the beginning of its eruption. The transverse velocities range from $40 \mathrm{~km} \mathrm{~s}^{-1}$ to $200 \mathrm{~km} \mathrm{~s}^{-1}$, very close to the values reported by Shen et al. (2011). The rotation slowed down from $\sim 122 \mathrm{~km} \mathrm{~s}^{-1}$ in the initial phase to $\sim 80 \mathrm{~km} \mathrm{~s}^{-1}$ in the later phase, which is in agreement with the cases of Liu et al. (2009) and Schmieder et al. (2013). The rotation, however, lasted for only one cycle with period of $\sim 7 \mathrm{~min}$,

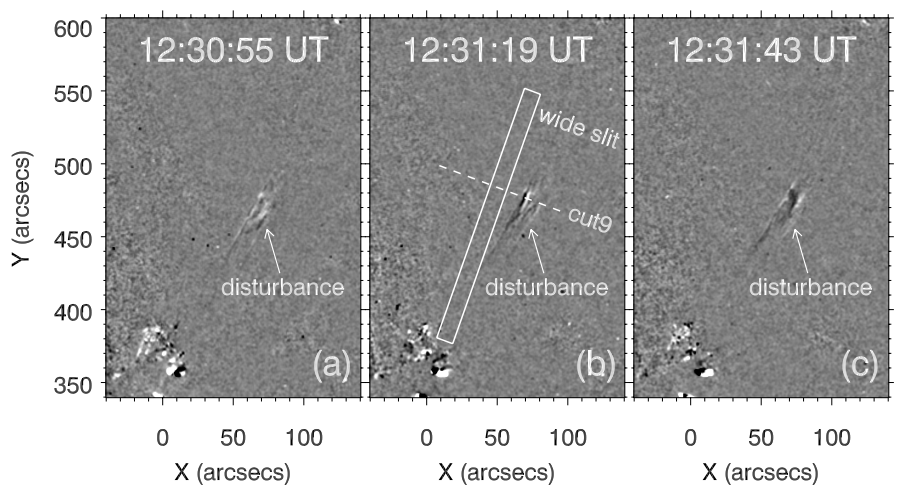

Fig. 11. Running-difference images in $193 \AA$ during 12:30-12:32 UT. White/black color denotes intensity increase/decrease. The arrows point to the transverse disturbances adjacent to the jet axis prior to the flare.

which is on the same order of magnitude as the previously reported values (Liu et al. 2009; Chen et al. 2012).

Precursor disturbances before the transverse jet rotation were observed. Figure 11 shows the running-difference images during 12:30-12:32 UT in $193 \AA$, where the white/black color represents intensity increase/decrease. Transverse disturbances adjacent to the jet axis are distinguishable in the images. In the time-slice diagram of cut9 (third row of Fig. 3), we label the disturbances with four dotted lines, the slopes of which represent the velocities, being $-175,126,-24$, and $51 \mathrm{~km} \mathrm{~s}^{-1}$, respectively. As to the cause of the disturbances, we noticed weak brightenings during 12:30-12:36 UT before the impulsive phase of the flare in the 0.4-5.0 $\AA$ light curve (Fig. 1d). Precursor brightenings, implying small-scale and weak release of magnetic energy prior to the impulsive phase of flares, have been observed (Harrison et al. 1985; Chifor et al. 2007), and are consistent with the mechanism of blowout jets (Moore et al. 2010).

In this paper, we report a flare-related jet observed by SDO/AIA on 2011 October 15. The jet underwent longitudinal rising/falling along its axis with an initial velocity of $254 \pm 10 \mathrm{~km} \mathrm{~s}^{-1}$. The effective gravitational acceleration was $-97 \pm 5 \mathrm{~m} \mathrm{~s}^{-2}$, well below the free-fall acceleration. The onset of jet eruption coincided with the beginning of the impulsive phase of the adjacent C1.6 flare. At the beginning of its longitudinal eruption, the jet presented helical structure and counterclockwise swirling motion that slowed down from an average of $\sim 122 \mathrm{~km} \mathrm{~s}^{-1}$ in the initial stage to $\sim 80 \mathrm{~km} \mathrm{~s}^{-1}$ in the later stage. The thick interwinding threads of the jet untwisted into multiple thin threads during the rotation that lasted for only one cycle with period of $\sim 7 \mathrm{~min}$ and amplitude that increases from $\sim 3.2 \mathrm{Mm}$ at the lower part to $\sim 11 \mathrm{Mm}$ at the upper part. Afterwards, a dimming region appeared following the curtainlike leading edge of the jet. The appearance/disappearance of dimming coincided with the ascending/descending motions. The cospatial EUV dimming and $\mathrm{H} \alpha$ surge indicate that the dimming resulted from the absorption of hot EUV emission by the cool surge. Line-of-sight mangetograms from SDO/HMI show that the flare/jet event was caused by continuous magnetic cancellation before the eruption. Potential-field extrapolation based on the magnetograms reveals that the jet was associated with the open magnetic fields at the boundary of AR 11314. The true height and velocity of the jet were estimated after considering the projection effect from the two perspectives of SDO and STEREO-B.

Acknowledgements. The authors are grateful to the referee for enlightening comments. Q.M.Z. acknowledges valuable discussions and suggestions with 
J. Q. Sun, P. F. Chen, Y. Guo, R. Erdélyi, G. Verth, Y. N. Su, and the solar physics group at the Purple Mountain Observatory. Q.M.Z. is also thankful to the Department of Applied Mathematics at the University of Sheffield for their hospitality during his visit. SDO is a mission of NASA's Living With a Star Program. AIA and HMI data are courtesy of the NASA/SDO science teams. STEREO/SECCHI data are provided by a consortium of US, UK, Germany, Belgium, and France. The Global Oscillation Network Group (GONG) Program is managed by the National Solar Observatory. This work is supported by Program 973 under grant 2011CB811402 and by NSFC 11303101, 11333009, 11173062 , and 11221063 .

\section{References}

Canfield, R. C., Reardon, K. P., Leka, K. D., et al. 1996, ApJ, 464, 1016 Chae, J., Qiu, J., Wang, H., \& Goode, P. R. 1999, ApJ, 513, L75 Chen, F., Ding, M. D., \& Chen, P. F. 2010, ApJ, 720, 1254

Chen, H. D., Zhang, J., \& Ma, S. L. 2012, Res. Astron. Astrophys., 12, 573 Chen, P. F. 2011, Liv. Rev. Sol. Phys., 8, 1

Chen, P. F., Wu, S. T., Shibata, K., \& Fang, C. 2002, ApJ, 572, L99

Cheng, X., Zhang, J., Liu, Y., \& Ding, M. D. 2011, ApJ, 732, L25

Chifor, C., Tripathi, D., Mason, H. E., \& Dennis, B. R. 2007, A\&A, 472, 967

Chifor, C., Isobe, H., Mason, H. E., et al. 2008, A\&A, 491, 279

Cirtain, J. W., Golub, L., Lundquist, L., et al. 2007, Science, 318, 1580

Culhane, L., Harra, L. K., Baker, D., et al. 2007, PASJ, 59, 751

De Pontieu, B., Erdélyi, R., \& James, S. P. 2004, Nature, 430, 536

Glesener, L., Krucker, S., \& Lin, R. P. 2012, ApJ, 754, 9

Guo, Y., Démoulin, P., Schmieder, B., et al. 2013, A\&A, 555, A19

Harrison, R. A., Waggett, P. W., Bentley, R. D., et al. 1985, Sol. Phys., 97, 387

Hong, J.-C., Jiang, Y.-C., Yang, J.-Y., et al. 2013, Res. Astron. Astrophys., 13, 253

Howard, R. A., Moses, J. D., Vourlidas, A., et al. 2008, Space Sci. Rev., 136, 67 Ji, H., Wang, H., Liu, C., \& Dennis, B. R. 2008, ApJ, 680, 734

Jiang, R.-L., Fang, C., \& Chen, P.-F. 2012, ApJ, 751, 152

Jiang, Y. C., Chen, H. D., Li, K. J., Shen, Y. D., \& Yang, L. H. 2007, A\&A, 469, 331

Jibben, P., \& Canfield, R. C. 2004, ApJ, 610, 1129

Kaiser, M. L. 2005, Adv. Space Res., 36, 1483

Kim, Y.-H., Moon, Y.-J., Park, Y.-D., et al. 2007, PASJ, 59, 763

Krucker, S., Kontar, E. P., Christe, S., Glesener, L., \& Lin, R. P. 2011, ApJ, 742, 82
Lee, K.-S., Innes, D. E., Moon, Y.-J., et al. 2013, ApJ, 766, 1

Lemen, J. R., Title, A. M., Akin, D. J., et al. 2012, Sol. Phys., 275, 17

Liu, C., Deng, N., Liu, R., et al. 2011a, ApJ, 735, L18

Liu, W., Berger, T. E., Title, A. M., \& Tarbell, T. D. 2009, ApJ, 707, L37

Liu, W., Berger, T. E., Title, A. M., Tarbell, T. D., \& Low, B. C. 2011b, ApJ, 728, 103

Liu, Y., \& Kurokawa, H. 2004, ApJ, 610, 1136

Moore, R. L., Cirtain, J. W., Sterling, A. C., \& Falconer, D. A. 2010, ApJ, 720, 757

Moreno-Insertis, F., \& Galsgaard, K. 2013, ApJ, 771, 20

Moreno-Insertis, F., Galsgaard, K., \& Ugarte-Urra, I. 2008, ApJ, 673, L211

Moschou, S. P., Tsinganos, K., Vourlidas, A., \& Archontis, V. 2012, Sol. Phys., 310

Nishizuka, N., Shimizu, M., Nakamura, T., et al. 2008, ApJ, 683, L83

Nisticò, G., Bothmer, V., Patsourakos, S., \& Zimbardo, G. 2009, Sol. Phys., 259, 87

Pariat, E., Antiochos, S. K., \& DeVore, C. R. 2009, ApJ, 691, 61

Pariat, E., Antiochos, S. K., \& DeVore, C. R. 2010, ApJ, 714, 1762

Patsourakos, S., Pariat, E., Vourlidas, A., Antiochos, S. K., \& Wuelser, J. P. 2008, ApJ, 680, L73

Pontin, D. I., Priest, E. R., \& Galsgaard, K. 2013, ApJ, 774, 154

Roy, J.-R. 1973, Sol. Phys., 32, 139

Savcheva, A., Cirtain, J., Deluca, E. E., et al. 2007, PASJ, 59, 771

Scherrer, P. H., Schou, J., Bush, R. I., et al. 2012, Sol. Phys., 275, 207

Schmieder, B., Shibata, K., van Driel-Gesztelyi, L., \& Freeland, S. 1995, Sol. Phys., 156, 245

Schmieder, B., Guo, Y., Moreno-Insertis, F., et al. 2013, A\&A, 559, A1

Shen, Y., Liu, Y., Su, J., \& Ibrahim, A. 2011, ApJ, 735, L43

Shibata, K., \& Uchida, Y. 1986, Sol. Phys., 103, 299

Shibata, K., Ishido, Y., Acton, L. W., et al. 1992, PASJ, 44, L173

Shibata, K., Nakamura, T., Matsumoto, T., et al. 2007, Science, 318, 1591

Shimojo, M., Hashimoto, S., Shibata, K., et al. 1996, PASJ, 48, 123

Singh, K. A. P., Isobe, H., Nishizuka, N., Nishida, K., \& Shibata, K. 2012, ApJ, 759,33

Török, T., Aulanier, G., Schmieder, B., Reeves, K. K., \& Golub, L. 2009, ApJ, 704,485

Wang, Y.-M., Sheeley, N. R., Jr., Socker, D. G., et al. 1998, ApJ, 508, 899

Yokoyama, T., \& Shibata, K. 1996, PASJ, 48, 353

Zhang, Q. M., \& Ji, H. S. 2013, A\&A, 557, L5

Zhang, J., Wang, J., \& Liu, Y. 2000, A\&A, 361, 759

Zhang, Q. M., Chen, P. F., Guo, Y., Fang, C., \& Ding, M. D. 2012, ApJ, 746, 19 